Random vibrations of stress-driven nonlocal beams with external damping

Preprint of the article published in Meccanica

DOI: $10.1007 / \mathrm{s} 11012-020-01181-7$

Francesco P. Pinnola,

Marzia S. Vaccaro Barretta,

Raffaele Barretta,

Francesco Marotti de Sciarra 


\title{
Random vibrations of stress-driven nonlocal beams with external damping
}

\author{
Francesco P. Pinnola - Marzia S. Vaccaro . \\ Raffaele Barretta - Francesco Marotti de \\ Sciarra
}

Received: date / Accepted: date

\begin{abstract}
Stochastic flexural vibrations of small-scale Bernoulli-Euler beams with external damping are investigated by stress-driven nonlocal mechanics. Damping effects are simulated considering viscous interactions between beam and surrounding environment. Loadings are modeled by accounting for their random nature. Such a dynamic problem is characterized by a stochastic partial differential equation in space and time governing time-evolution of the relevant displacement field. Differential eigenanalyses are performed to evaluate modal time coordinates and mode shapes, providing a complete stochastic description of response solutions. Closed-form expressions of power spectral density, correlation function, stationary and non-stationary variances of displacement fields are analytically detected. Size-dependent dynamic behaviour is assessed in terms of stiffness, variance and power spectral density of displacements. The outcomes can be useful for design and optimization of structural components of modern small-scale devices, such as Micro- and Nano-Electro-Mechanical-Systems (MEMS and NEMS).
\end{abstract}

Keywords Stochastic dynamics · small-scale beams · size effects · viscous damping $\cdot$ stress-driven nonlocal integral elasticity $\cdot$ MEMS/NEMS

\section{Literature survey, motivation and outline}

Methodologies to predict random vibrations in structural systems have reached over the last century a significant importance in design and optimization of newgeneration composites [1, 2] and technological devices, such as: micro-bridges [3], nano-switches [4], nano-generators [5, 6], nano-sensors [7, 8], energy harvesters [911]. It is acknowledged that continuum mechanics is able to model structural components of small-scale systems [12], but some mechanical expedients are needed to accurately predict nonconventional phenomena, such as size and damping effects.

F.P. Pinnola · M.S. Vaccaro - R. Barretta - F. Marotti de Sciarra Department of Structures for Engineering and Architecture, University of Naples Federico II,

via Claudio 21, Ed. 6, 80125 - Naples, Italy

E-mail: rabarret@unina.it 
It is well-established that inter-atomic forces and molecular interactions cannot be overlooked in small-scale structures which exhibit technically significant size effects [13-18]. Such a phenomenon cannot be captured by the classical theory of local continuum [19] due to lack of internal characteristic scales. Nonlocal continua, driven by suitably chosen scale characteristic parameters, are instead appropriate to model micro- and nano-structures [20-23], as confirmed by molecular dynamic simulations [24-26]. Nonlocal methodologies allow for modeling complex mechanical behaviours avoiding computationally expensive procedures [27, 28].

Nonlocal theory, in its earliest formulation [29, 30] is based on the idea that the stress at a point of a continuum depends not only on elastic strain at that point but involves local responses of the whole structure. Long-range interactions are thus described by a strain-driven convolution integral supplemented with an averaging kernel characterized by a nonlocal length-scale parameter. Such an approach was consistently applied by Eringen [31, 32] to nonlocal problems involving screw dislocation and wave propagation, that are formulated in unbounded domains. However, mathematical difficulties are apparent when the strain-driven strategy is applied to structural problems which are generally defined in bounded domains.

In fact, assuming that the averaging kernel is the Green function of a differential operator, from the integral convolution it is possible to obtain a consequent differential formulation [33, 34]. For bounded structural domains, to the differential formulation above is needed to add a proper set of Constitutive Boundary Conditions (CBCs) [35]. Paradoxical results and unacceptable nonlocal responses are obtained $[36,37]$ if CBCs are ignored. Several nonlocal theories have been adopted to overcome the aforementioned difficulties, such as: two-phase models $[38,39]$, strain and stress gradient theories [40-44], nonlocal gradient techniques [45-47], strain-difference approaches [48-50], displacement-based nonlocal models [51-54], stress-driven formulation of nonlocality [55, 56]. Advantageously, the stress-driven approach has been shown to be able to effectively model the nonlocal behaviour of small-scale structures and provides exact solutions for problems of applicative interest in Nano-Engineering [57, 58]. The stress-driven nonlocal integral model is therefore adopted in this paper to significantly tackle size-dependent random vibrations in inflected elastic small-scale beams. A first effort on the matter, disregarding random phenomena, was performed in [59].

Another import effect in dynamics of micro- and nano-systems concerns damping phenomena which should be properly modeled in applicative problems of nanoengineering. Instances are listed as follows: external magnetic force [60], humidity, thermal and paddling effects [61] and internal viscous force due to material rheological properties [62]. Modeling of internal viscous forces is successfully performed by properly selecting constitutive formulations [63]. Other effects, mentioned above, are related to surrounding environmental interactions [64]. In the present paper, particular attention is paid to capture external damping effects, useful to analyse modern small-scale structures, such as: sensors inside viscous fluid, devices under magnetic field, nano-systems for biological detection. Specifically, a bed of independent dashpots will be considered to simulate external viscous interactions between nonlocal beam and surrounding environment.

At micro- and nano-scales, structures can be excited by different kind of force systems. An example is the effect of environmental thermal and/or mechanical noises in nano-sensors [65]. Stochastic approaches can be conveniently exploited to 
model external loadings [66-68], effectively representable by random time-process [69-71].

For the aforesaid reasons, the present research provides a novel strategy for stress-driven nonlocal analysis of damped vibrations of elastic nano-beams due to stochastic excitation. Steady-state solutions are established, detecting thus analytical expressions of power spectral density and stationary variance of displacements. Closed-form solutions are also evaluated for non-stationary responses of nonlocal damped beams forced by Gaussian white noise.

The manuscript is organized as follows. Strain- and stress-driven models of pure nonlocal integral elasticity are recalled and specialized to Bernoulli-Euler beams in Section 2. Dynamic equilibrium equations of damped beams are established in Section 3 by using the well-posed stress-driven nonlocal strategy of elasticity. Mode shape functions and natural frequencies are analytically detected and an effective methodology to perform dynamic eigenanalysis is also elucidated. A stochastic analysis of nonlocal damped beams forced by Gaussian white noise is developed in Section 4. Both stationary and non-stationary examinations are performed, detecting thus closed form solutions of displacement variance, power spectral density and correlation function. Numerical simulations are implemented in Section 5 to test accuracy of obtained solutions. Analytical stationary and non-stationary variances are compared with numerical outcomes derived by Monte Carlo simulations. A parametric study of stochastic responses, in terms of displacement variances and natural frequencies, is given in Section 5 to study nonlocal effects. Closing remarks are outlined in Section 6.

\section{Purely nonlocal integral elasticity}

Two purely nonlocal models of elasticity are available in literature:

1. strain-driven integral law [29], applied to problems of screw dislocation and wave propagation [31, 32];

2. stress-driven integral law [55] applied to nonlocal mechanics of structures.

These theories are preliminarily recalled below for 3-D continua and specialized to Bernoulli-Euler beams. Eringen's strain-driven law is based on the idea that the stress $\boldsymbol{\sigma}$ at a point $\boldsymbol{x}$ of a nonlocal 3 -D continuous body $\mathcal{B}$ is the output of a convolution between the local response to the elastic strain field $\varepsilon$ and a scalar kernel $\Phi_{\lambda}$ depending on a non-dimensional positive nonlocal parameter $\lambda$. That is,

$$
\boldsymbol{\sigma}(\boldsymbol{x})=\int_{\mathcal{B}} \Phi_{\lambda}(\boldsymbol{x}, \overline{\boldsymbol{x}}) \boldsymbol{E}(\overline{\boldsymbol{x}}) \varepsilon(\overline{\boldsymbol{x}}) d \overline{\boldsymbol{x}}
$$

with $\boldsymbol{E}$ fourth-order local elasticity stiffness tensor.

For a Bernoulli-Euler inflected beam of length $L$, the nonlocal strain-driven relation Eq. (1) takes the form

$$
M(z)=E I \int_{0}^{L} \Phi_{\lambda}(z, \bar{z}) \chi(\bar{z}) d \bar{z}
$$

with $M$ bending moment, $E$ Euler-Young modulus, $I$ cross-sectional moment of inertia along the bending axis $y, z$ beam axial abscissa and $\chi$ elastic curvature. 
The integral kernel $\Phi_{\lambda}$ can be selected among exponential, Gaussian or powerlaw type functions and must satisfy properties of positivity, symmetry and limit impulsivity [32]. A convenient choice for the averaging kernel is the special biexponential function

$$
\Phi_{\lambda}(z, \bar{z})=\frac{1}{2 \lambda L} \exp \left(-\frac{|z-\bar{z}|}{\lambda L}\right)
$$

where $\lambda L$ is the characteristic length $L_{c}$. With the assumption above, the integral law Eq. (2) is equivalent to the second-order differential equation [35]

$$
M^{(2)}(z)-\frac{1}{(\lambda L)^{2}} M(z)=-\frac{E I}{(\lambda L)^{2}} \chi(z)
$$

supplemented with the following constitutive boundary conditions (CBC)

$$
\left\{\begin{array}{l}
M^{(1)}(0)=\frac{1}{\lambda L} M(0) \\
M^{(1)}(L)=-\frac{1}{\lambda L} M(L)
\end{array}\right.
$$

It is worth noting that, for structural problems of applicative interest, CBCs Eq. (5) are in contrast with equilibrium requirements [35]. Incompatibility between equilibrium and constitutive conditions reveals that Eringen's nonlocal model leads to ill-posed structural problems, generally formulated in bounded domains. Such an obstruction can be overcome by using the stress-driven approach [55] which is well-posed. Exact nonlocal structural solutions can be found in [58].

In stress-driven mechanics, the elastic strain $\varepsilon$ at a point $\boldsymbol{x}$ of $\mathcal{B}$ is obtained by convoluting the local stress $\boldsymbol{\sigma}$ with an averaging kernel $\Phi_{\lambda}$

$$
\varepsilon(\boldsymbol{x})=\int_{\mathcal{B}} \Phi_{\lambda}(\boldsymbol{x}, \overline{\boldsymbol{x}}) \boldsymbol{C}(\overline{\boldsymbol{x}}) \boldsymbol{\sigma}(\overline{\boldsymbol{x}}) d \overline{\boldsymbol{x}}
$$

with $\boldsymbol{C}=\boldsymbol{E}^{-1}$ local elastic compliance. The stress-driven model for BernoulliEuler beams is governed by the following moment-curvature relation

$$
\chi(z)=\frac{1}{E I} \int_{0}^{L} \Phi_{\lambda}(z-\bar{z}) M(\bar{z}) d \bar{z}
$$

The integral formulation Eq. (7), with the special kernel Eq. (3), is equivalent to the second order differential equation [55]

$$
\chi^{(2)}(z)-\frac{1}{(\lambda L)^{2}} \chi(z)=-\frac{1}{E I(\lambda L)^{2}} M(z)
$$

equipped with the following constitutive boundary conditions

$$
\left\{\begin{array}{l}
\chi^{(1)}(0)=\frac{1}{\lambda L} \chi(0) \\
\chi^{(1)}(L)=-\frac{1}{\lambda L} \chi(L)
\end{array}\right.
$$

The stress-driven approach Eqs. (8), (9) provides exact solutions in both static and dynamic structural problems and is exploited in the present study to analytically tackle size-dependent random vibrations of slender elastic beams. 


\section{Dynamical analysis of nonlocal beams}

Let us consider a nonlocal beam of length $L$ and cross-sectionial area $A_{t}$ subjected to a transverse loading per unit of length $q(z, t)$, see Fig. $1 .(x, y, z)$ is the adopted system of orthogonal coordinates, $v(z, t)$ denotes the transverse displacement and $\rho(z)$ is the mass density. The stress-driven formulation Eqs. (8), (9) is used as

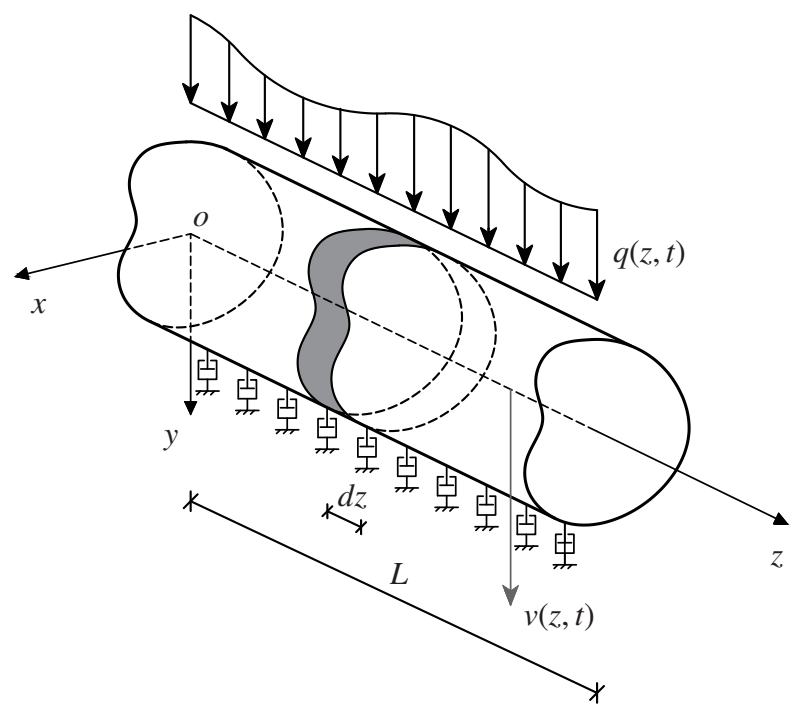

Fig. 1: Bernoulli-Euler beam with external damping

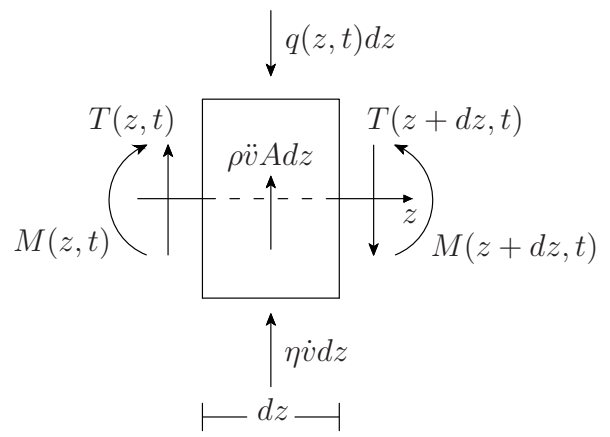

Fig. 2: Free-body diagram of a beam differential element

nonlocal elasticy law while the effect of external damping is introduced and modeled as a bed of dashpots with viscosity $\eta$. This kind of viscous interaction is able to model a possible external interaction between nonlocal beam and viscous fluid. Damping can be also simulated as a material effect [72-77] by a viscoelastic law. 
In this paper, following the Newtonian approach, we consider a beam differential element of length $d z$, whose free-body diagram is represented in Fig. 2. The equilibrium equation along the $y$-direction, involving external loading, inertial and damping forces, and bending and shearing fields, writes as

$$
\frac{\partial T(z, t)}{\partial z} d z+q(z, t) d z=\eta d z \frac{\partial v(z, t)}{\partial t}+\rho A d z \frac{\partial^{2} v(z, t)}{\partial t^{2}}, \quad 0<z<L
$$

By ignoring second-order terms in $d z$ and neglecting mass moment of inertia and angular acceleration, the rotational equilibrium along the $x$-axis gives

$$
\frac{\partial M(z, t)}{\partial z}-T(z, t)=0, \quad 0<z<L
$$

Combining the equilibrium equations along $x$ - and $y$-directions we get the partial differential equation

$$
-\frac{\partial^{2} M(z, t)}{\partial z^{2}}+\eta \frac{\partial v(z, t)}{\partial t}+\rho A \frac{\partial^{2} v(z, t)}{\partial t^{2}}=q(z, t), \quad 0<z<L
$$

By introducing the nonlocal stress-driven relation in Eq. (8), from Eq. (12) we get

$$
-E I\left[\frac{\partial^{2} \chi(z, t)}{\partial z^{2}}-(\lambda L)^{2} \frac{\partial^{4} \chi(z, t)}{\partial z^{4}}\right]+\eta \frac{\partial v(z, t)}{\partial t}+\rho A \frac{\partial^{2} v(z, t)}{\partial t^{2}}=q(z, t)
$$

According to Bernoulli-Euler kinematics, curvature $\chi(z, t)$ is related to the transverse displacement $v(z, t)$ by

$$
\chi(z, t)=-\frac{\partial^{2} v(z, t)}{\partial z^{2}}
$$

and by placing Eq. (14) into Eq. (13) we have that

$$
\frac{\partial^{4} v(z, t)}{\partial z^{4}}-(\lambda L)^{2} \frac{\partial^{6} v(z, t)}{\partial z^{6}}+\frac{\eta}{E I} \frac{\partial v(z, t)}{\partial t}+\frac{\rho A}{E I} \frac{\partial^{2} v(z, t)}{\partial t^{2}}=\frac{q(z, t)}{E I}
$$

which is the partial differential equilibrium equation ruling bending vibrations of a nonlocal Bernoulli-Euler beam resting on a bed of dashpots.

For vanishing nonlocal parameter $\lambda \rightarrow 0^{+}$, Eq. (15) provides the known differential equation governing forced vibrations of local Bernoulli-Euler beams resting on a bed of independent dashpots. Moreover, setting $\eta=0$, the classical formulation of undamped local beams is obtained [78, 79]. Solutions of the introduced partial differential equation may be found by imposing two initial conditions, four standard BCs and two constitutive BCs of the stress-driven model Eq. (9). 
3.1 Free vibrations of undamped nonlocal beam

In order to solve Eq. (15), we first consider the undamped nonlocal beam in free vibration. In other words, we set $q(z, t)=0$ and $\eta=0$. These assumptions imply that Eq. (15) yields

$$
\frac{\partial^{4} v(z, t)}{\partial z^{4}}-(\lambda L)^{2} \frac{\partial^{6} v(z, t)}{\partial z^{6}}+\frac{\rho A}{E I} \frac{\partial^{2} v(z, t)}{\partial t^{2}}=0, \quad 0<z<L
$$

which rules free vibrations of undamped nonlocal beam. We suppose that the displacement function $v(z, t)$ is with separable variables and so it can be expressed by a product of a space function $\phi(z)$ (mode shape) and a time-dependent function $y(t)$ that modulates the amplitude of mode shape in time. That is,

$$
v(z, t)=\phi(z) y(t)
$$

by substituting Eq. (17) into Eq. (16) we get

$$
y(t)\left[\frac{d^{4} \phi(z)}{d z^{4}}-(\lambda L)^{2} \frac{d^{6} \phi(z)}{d z^{6}}\right]+\frac{\rho A}{E I} \phi(z) \frac{d^{2} y(t)}{d t^{2}}=0, \quad 0<z<L
$$

where partial derivatives have been replaced by total derivatives due to the assumption in Eq. (17). Moreover, by using Lagrange's differential notation for space derivative and Newton's notation for time derivative we can rewrite Eq. (18) as

$$
\frac{E I}{\rho A} \frac{(\lambda L)^{2} \phi^{(6)}(z)-\phi^{(4)}(z)}{\phi(z)}=\frac{\ddot{y}(t)}{y(t)}, \quad 0<z<L
$$

where both sides of Eq. (19) must be equal to a constant $\alpha$ that can be associated with the natural frequency of the oscillation $\omega_{0}$ as

$$
\alpha=-\omega_{0}^{2}
$$

Hence, Eq. (19) can be rewritten as

$$
\frac{E I}{\rho A} \frac{(\lambda L)^{2} \phi^{(6)}(z)-\phi^{(4)}(z)}{\phi(z)}=\frac{\ddot{y}(t)}{y(t)}=-\omega_{0}^{2}
$$

by considering the left side of Eq. (21), we get the following sixth order differential equation in the space variable $z$

$$
(\lambda L)^{2} \phi^{(6)}(z)-\phi^{(4)}(z)+\omega_{0}^{2} \frac{\rho A}{E I} \phi(z)=0, \quad 0<z<L
$$

whose solution must satisfy the four BCs depending on the type of loads and constraints at the bounds and the two constitutive BCs in Eq. (9). That is,

$$
\left\{\begin{array}{l}
\phi^{(3)}(0)=\frac{1}{\lambda L} \phi^{(2)}(0) \\
\phi^{(3)}(L)=-\frac{1}{\lambda L} \phi^{(2)}(L)
\end{array}\right.
$$

The problem to find the constant $\omega_{0}$ and $\phi(z)$ such that Eq. (22) admits nontrivial solution is known as differential eigenvalue-eigenfunction problem, where $\omega_{0}$ is the eigenvalue and $\phi(z)$ represents the corresponding eigenfunction. Eq. (22) 
admits infinite eigenvalues and then infinite eigenfunctions. Therefore, the solution in terms of displacement can be expressed as a sum of infinite products between the modal time coordinates $y_{j}(t)$ and the mode shapes $\phi_{j}(z)$

$$
v(z, t)=\sum_{j=1}^{\infty} \phi_{j}(z) y_{j}(t)
$$

where the $j$-th eigenfunction $\phi_{j}(z)$ is a solution of the $j$-th homogeneous sixthorder differential equation in Eq. (22). Specifically,

$$
\phi_{j}(z)=\sum_{i=1}^{3}\left\{C_{i} \exp \left[\sqrt{\gamma_{i}\left(\omega_{0, j}\right)} z\right]+C_{i+3} \exp \left[-\sqrt{\gamma_{i}\left(\omega_{0, j}\right)} z\right]\right\}
$$

where $\gamma_{i}\left(\omega_{0, j}\right)$ with $i=1,2,3$ are the roots of the following characteristic third degree polynomial

$$
(\lambda L)^{2} \gamma^{3}-\gamma^{2}+\omega_{0, j}^{2} \frac{\rho A}{E I}=0
$$

and the coefficients $C_{i}$ are obtained by imposing that solution in Eq. (25) satisfies the previous six BCs and the normality condition. In this manner, each eigenfunction possesses the following orthonormality property

$$
\int_{0}^{L} \phi_{i}(z) \phi_{j}(z) d z=\delta_{k j}
$$

where $\delta_{k j}$ indicates the Kronecker delta defined as

$$
\delta_{k j}= \begin{cases}1 & \text { if } k=j \\ 0 & \text { if } k \neq j\end{cases}
$$

Such solution of differential eigenvalue problem will be used in the next section to solve the more general case of a forced nonlocal damped beam.

3.2 Forced vibrations of damped nonlocal beam

Now, we consider the dynamical problem of a forced beam with external damping. The solution in terms of displacements expressed in Eq. (24) in function of mode shapes $\phi_{j}(z)$ is placed into the equilibrium equation (15). Specifically,

$$
\begin{aligned}
& \frac{E I}{\rho A}\left[\sum_{j=1}^{\infty} y_{j}(t) \phi_{j}^{(4)}(z)-\sum_{j=1}^{\infty} y_{j}(t)(\lambda L)^{2} \phi_{j}^{(6)}(z)\right]+ \\
& +\frac{\eta}{\rho A} \sum_{j=1}^{\infty} \dot{y}_{j}(t) \phi_{j}(z)+\sum_{j=1}^{\infty} \ddot{y}_{j}(t) \phi_{j}(z)=\frac{q(z, t)}{\rho A}
\end{aligned}
$$


by multiplying both sides of Eq. (29) by the $i$-th eigenfunction $\phi_{i}(z)$ and integrating on the domain $[0, L]$, Eq. (29), we get

$$
\begin{aligned}
& \frac{E I}{\rho A}\left[\sum_{j=1}^{\infty} y_{j}(t) \int_{0}^{L} \phi_{i}(z) \phi_{j}^{(4)}(z) d z\right]+ \\
& -\frac{E I}{\rho A}\left[\sum_{j=1}^{\infty} y_{j}(t)(\lambda L)^{2} \int_{0}^{L} \phi_{i}(z) \phi_{j}^{(6)}(z) d z\right]+ \\
& +\frac{\eta}{\rho A} \sum_{j=1}^{\infty} \dot{y}_{j}(t) \int_{0}^{L} \phi_{i}(z) \phi_{j}(z) d z+\sum_{j=1}^{\infty} \ddot{y}_{j}(t) \int_{0}^{L} \phi_{i}(z) \phi_{j}(z) d z= \\
& \frac{1}{\rho A} \int_{0}^{L} \phi_{i}(z) q(z, t) d z
\end{aligned}
$$

Taking into account the eigenfunctions orthonormality property in Eq. (27), from Eq. (30) we get the second order differential equation in terms of modal coordinate $y_{i}(t)$ that rules the motion of a forced damped modal oscillator

$$
\frac{E I}{\rho A} y_{i}(t)\left[a_{i}-(\lambda L)^{2} b_{i}\right]+\frac{\eta}{\rho A} \dot{y}_{i}(t)+\ddot{y}_{i}(t)=\frac{1}{\rho A} \int_{0}^{L} \phi_{i}(z) q(z, t) d z
$$

where the coefficients $a_{i}$ and $b_{i}$ are

$$
a_{i}=\int_{0}^{L} \phi_{i}(z) \phi_{i}^{(4)}(z) d z, \quad b_{i}=\int_{0}^{L} \phi_{i}(z) \phi_{i}^{(6)}(z) d z
$$

Moreover, we assume that also the load is with separable variables. Therefore,

$$
q(z, t)=g(z) f(t)
$$

this implies that Eq. (31) can be rewritten as

$$
\frac{k_{\lambda, i}}{\rho A} y_{i}(t)+\frac{\eta}{\rho A} \dot{y}_{i}(t)+\ddot{y}_{i}(t)=\frac{c_{i}}{\rho A} f(t)
$$

where the nonlocal modal stiffness $k_{\lambda, i}$ is defined as

$$
k_{\lambda, i}=E I\left[a_{i}-(\lambda L)^{2} b_{i}\right]
$$

and the coefficient $c_{i}$ is

$$
c_{i}=\int_{0}^{L} \phi_{i}(z) g(z) d z
$$

Notice that the following term

$$
\omega_{0, i}=\sqrt{\frac{k_{\lambda, i}}{\rho A}}
$$

represents the natural frequency associated to the $i$-th mode $\phi_{j}(z)$.

Solution of Eq. (34) provides the $i$-th modal time coordinate of the infinite series in Eq. (24). Truncating the sum to an appropriate number $n$ of eigenfunctions and modal coordinates leads to the following approximated solution, that will be used for the numerical applications

$$
v(z, t) \approx \sum_{j=1}^{n} \phi_{j}(z) y_{j}(t)
$$




\section{Stochastic analysis of nonlocal beams}

Now we turn the attention to random vibrations of nonlocal beams, supposing that the time-dependent part of the transverse load $q(z, t)$ has a stochastic nature. Specifically, from Eq. (33) we assume that

$$
q(z, t)=g(z) F(t)
$$

where $g(z)$ is a deterministic function, while $F(t)$ is a stochastic process, the latter is noted by a capital letter to distinguish it from the deterministic function $f(t)$. Moreover, we assume that $F(t)$ is a stationary Gaussian process with zero mean $\mu_{F}$ and with an assigned correlation function $(\mathrm{CF})$ denoted by $R_{F}(\tau)$. Thus, the input process is completely described by the following time-independent parameters

$$
\begin{aligned}
\mu_{F}=\mu_{F}(t) & :=\mathbb{E}[F(t)]=0 \\
R_{F}(\tau)=R_{F}(t, t+\tau): & =\mathbb{E}[F(t) F(t+\tau)]-\mu_{F}^{2} \\
& =\mathbb{E}[F(t) F(t+\tau)]
\end{aligned}
$$

where $\mathbb{E}[\cdot]$ is the averaging operator. For $\tau=0$ the $\mathrm{CF}$ gives the value of the variance $\sigma_{F}^{2}$. That is,

$$
\sigma_{F}^{2}=\mathbb{E}[F(t) F(t)]=R_{F}(0)
$$

Being $F(t)$ a stationary process, by virtue of Wiener-Khinchin theorem, the power spectral density (PSD), denoted by $S_{F}(\omega)$, is the Fourier transform of the correlation function. That is,

$$
\begin{aligned}
S_{F}(\omega): & =\frac{1}{2 \pi} \int_{-\infty}^{\infty} R_{f}(\tau) e^{-\mathrm{i} \omega \tau} d \tau \\
& =\lim _{\mathrm{T} \rightarrow \infty} \frac{1}{2 \pi \mathrm{T}} \mathbb{E}\left[\hat{F}^{*}(\omega, \mathrm{T}) \hat{F}(\omega, \mathrm{T})\right]
\end{aligned}
$$

where $\mathrm{i}=\sqrt{-1}$ is the imaginary unit, $\hat{F}(\omega, \mathrm{T})$ indicates the truncated Fourier transform of the process $F(t)$ in a finite time interval $[0, \mathrm{~T}]$, and $\hat{F}^{*}(\omega, \mathrm{T})$ denotes its complex conjugate.

By taking into account Eq. (39) and the definition in Eq. (40b), CF of the loading $q(z, t)$ is given by

$$
\begin{aligned}
R_{q}(z, \tau) & =\mathbb{E}[q(z, t) q(z, t+\tau)] \\
& =g(z) \mathbb{E}[F(t) F(t+\tau)] g(z) \\
& =g^{2}(z) R_{F}(\tau)
\end{aligned}
$$

Similarly, the PSD of the loading is

$$
\begin{aligned}
S_{q}(z, \omega) & =\lim _{\mathrm{T} \rightarrow \infty} \frac{1}{2 \pi \mathrm{T}} \mathbb{E}\left[\hat{q}^{*}(z, \omega, \mathrm{T}) \hat{q}(z, \omega, \mathrm{T})\right] \\
& =g(z) \lim _{\mathrm{T} \rightarrow \infty} \frac{1}{2 \pi \mathrm{T}} \mathbb{E}\left[\hat{F}^{*}(\omega, \mathrm{T}) \hat{F}(\omega, \mathrm{T})\right] g(z) \\
& =g^{2}(z) S_{F}(\omega)
\end{aligned}
$$

and the variance $\sigma_{q}^{2}(z)$ is

$$
\sigma_{q}^{2}(z)=\mathbb{E}[q(z, t) q(z, t)]=g(z) \sigma_{F}^{2} g(z)
$$


Now we want to characterize the response process in terms of displacement $v(z, t)$ when the input is a Gaussian stationary process. Being the stochastic input Gaussian also the stochastic output will be Gaussian, but the response process will have a stationary part for $t \gg 0$ and a transient non-stationary one. Therefore, it is needed the evaluation of the evolution in time of the statistics.

\subsection{Time-domain response}

By observing Eq. (34) we deduce that if the forcing load $f(t)=F(t)$, then also the time response of the beam in terms of modal coordinate will be a stochastic process $Y(t)$. Hence, from Eq. (24) we get

$$
v(z, t)=\sum_{j=1}^{\infty} \phi_{j}(z) Y_{j}(t)
$$

where $\phi_{j}(z)$ is a deterministic function that can be analytically evaluated. While, the stochastic response $Y_{j}(t)$ is solution of the following stochastic differential equation

$$
\ddot{Y}_{j}(t)+\frac{\eta}{\rho A} \dot{Y}_{j}(t)+\frac{k_{\lambda, j}}{\rho A} Y_{j}(t)=\frac{c_{j}}{\rho A} F(t)
$$

The forced input $F(t)$ is a Gaussian process and the differential equation in Eq. (47) is linear. This implies that the output process $Y_{j}(t)$ will be Gaussian too, and then can be completely characterized by the mean $\mu_{Y_{j}}(t)$ and the correlation function $R_{Y_{j}}(t, t+\tau)$. Under the assumption that the beam is quiescent at $t=0$, the two initial conditions become

$$
\left\{\begin{array}{l}
v(z, 0)=0, \forall z \in[0, L] \Rightarrow Y_{j}(0)=0, \forall j \in \mathbb{N}^{+} \\
\dot{v}(z, 0)=0, \forall z \in[0, L] \Rightarrow \dot{Y}_{j}(0)=0, \forall j \in \mathbb{N}^{+}
\end{array}\right.
$$

The output process is obtained by applying the Duhamel superposition integral

$$
Y_{j}(t)=\frac{c_{j}}{\rho A} \int_{0}^{t} h_{j}(t-\tau) F(\tau) d \tau
$$

being $h_{j}(t)$ a deterministic function which represents the impulse response of the $j$-th modal oscillator. Such a function is defined as

$$
h_{j}(t)=\sqrt{\frac{4(\rho A)^{2}}{4 k_{\lambda, j \rho A}-\eta^{2}}} \exp \left(-\frac{\eta}{2 \rho A} t\right) \sin \left(\sqrt{\frac{4 k_{\lambda, j} \rho A-\eta^{2}}{4(\rho A)^{2}}} t\right)
$$

We can observe that the term

$$
\omega_{D, j}=\sqrt{\frac{4 k_{\lambda, j} \rho A-\eta^{2}}{4(\rho A)^{2}}}
$$

is the damped frequency. Therefore, the impulse response can be rewritten as

$$
h_{j}(t)=\frac{1}{\omega_{D, j}} \exp \left(-\frac{\eta}{2 \rho A} t\right) \sin \left(\omega_{D, j} t\right)
$$


Taking Eq. (40a) into account and by applying the averaging operator to Eq. (49) we can prove that the mean of the response process is zero. That is,

$$
\begin{aligned}
\mu_{Y_{j}}(t) & =\mathbb{E}\left[\frac{c_{j}}{\rho A} \int_{0}^{t} h_{j}(t-\tau) F(\tau) d \tau\right] \\
& =\frac{c_{j}}{\rho A} \int_{0}^{t} h_{j}(t-\tau) \mathbb{E}[F(\tau)] d \tau \\
& =\frac{c_{j}}{\rho A} \int_{0}^{t} h_{j}(t-\tau) \mu_{F}(\tau) d \tau=0
\end{aligned}
$$

Eq. (53) implies that also the mean of displacements is zero $\mu_{v}(z, t)=0$ for all $t \geqslant 0$, and for $z \in[0, L]$.

$\mathrm{CF}$ of the response process $Y_{j}(t)$ can be evaluated taking into account the assumption in Eq. (40b) and the Duhamel integral in Eq. (49). We apply the averaging operator to the process $Y_{j}(t)$ considering two different time-step $t=t_{1}$ and $t+\tau=t_{2}$. That is,

$$
\begin{aligned}
R_{Y_{j}}\left(t_{1}, t_{2}\right): & =\mathbb{E}\left[Y_{j}\left(t_{1}\right) Y_{j}\left(t_{2}\right)\right] \\
& =\frac{c_{j}^{2}}{(\rho A)^{2}} \int_{0}^{t_{1}} \int_{0}^{t_{2}} h_{j}\left(t_{1}-\tau_{1}\right) h_{j}\left(t_{2}-\tau_{2}\right) \mathbb{E}\left[F\left(\tau_{1}\right) F\left(\tau_{2}\right)\right] d \tau_{1} d \tau_{2} \\
& =\frac{c_{j}^{2}}{(\rho A)^{2}} \int_{0}^{t_{1}} \int_{0}^{t_{2}} h_{j}\left(t_{1}-\tau_{1}\right) h_{j}\left(t_{2}-\tau_{2}\right) R_{F}\left(\tau_{2}-\tau_{1}\right) d \tau_{1} d \tau_{2}
\end{aligned}
$$

from Eq. (54) the variance $\sigma_{Y_{j}}^{2}(t)$ can be also evaluated by placing $t_{1}=t_{2}$.

By taking into account the Eq. (46) the CF of the displacement $v(z, t)$ is

$$
\begin{aligned}
R_{v}\left(z, t_{1}, t_{2}\right): & =\mathbb{E}\left[v\left(z, t_{1}\right) v\left(z, t_{2}\right)\right] \\
& =\sum_{j=1}^{\infty} \sum_{i=1}^{\infty} \phi_{j}(z) R_{Y_{j} Y_{i}}\left(t_{1}, t_{2}\right) \phi_{i}(z)
\end{aligned}
$$

where $R_{Y_{j} Y_{i}}\left(t_{1}, t_{2}\right)$ is the cross correlation of the modal response processes $Y_{j}(t)$ and $Y_{i}(t)$ defined as

$$
\begin{aligned}
R_{Y_{j} Y_{i}}\left(t_{1}, t_{2}\right): & =\mathbb{E}\left[Y_{j}\left(t_{1}\right) Y_{i}\left(t_{2}\right)\right] \\
& =\frac{c_{j} c_{i}}{(\rho A)^{2}} \int_{0}^{t_{1}} \int_{0}^{t_{2}} h_{j}\left(t_{1}-\tau_{1}\right) h_{i}\left(t_{2}-\tau_{2}\right) R_{F}\left(\tau_{2}-\tau_{1}\right) d \tau_{1} d \tau_{2}
\end{aligned}
$$

If $t_{1}=t_{2}=t$ Eq. (55) provides the non-stationary variance of the displacement $v(z, t)$. That is,

$$
\begin{aligned}
\sigma_{v}^{2}(z, t): & =\mathbb{E}[v(z, t) v(z, t)] \\
& =\sum_{j=1}^{\infty} \sum_{i=1}^{\infty} \phi_{j}(z) \sigma_{Y_{j} Y_{i}}^{2}(t) \phi_{i}(z)
\end{aligned}
$$


where $\sigma_{Y_{j} Y_{i}}^{2}(t)$ is

$$
\begin{aligned}
\sigma_{Y_{j} Y_{i}}^{2}(t): & =\mathbb{E}\left[Y_{j}(t) Y_{i}(t)\right] \\
& =\frac{c_{j} c_{i} \sigma_{F}^{2}}{(\rho A)^{2}} \int_{0}^{t} \int_{0}^{t} h_{j}(t-\tau) h_{i}(t-\tau) d \tau d \tau \\
& =\frac{c_{j} c_{i} \sigma_{F}^{2}}{(\rho A)^{2}} t \int_{0}^{t} h_{j}(t-\tau) h_{i}(t-\tau) d \tau
\end{aligned}
$$

and represents the cross variance of the modal response processes $Y_{j}(t)$ and $Y_{i}(t)$.

\subsubsection{Monte Carlo simulation}

In some cases, Eq. (56) and Eq. (58) cannot be evaluated in closed form and it is needed a numerical approach to characterized the response process form a stochastic point of view. In this context, Monte Carlo (MC) method is a powerful tool that provides a time-domain response with the aid of digital simulations. Specifically, as a first step, it is needed the generation of a proper number $N$ of samples (or realizations) of the stochastic input $F(t)$. The $i$-th realization of the stochastic input process is denoted as $F^{i}(t)$ and can be generated by harmonic superposition method proposed by Shinozuka and Deodatis [80]. According to this approach the generic $i$-th sample of the forced process is given as

$$
F^{i}(t)=\sqrt{2} \sum_{j=1}^{m} \sqrt{2 S_{F}\left(\omega_{j}\right) \Delta \omega} \cos \left(\omega_{j} t+\theta_{j}^{i}\right)
$$

where $\omega_{j}=j \Delta \omega, \Delta \omega$ is the discretization step in the frequency domain of the PSD function $S_{F}(\omega), \theta_{j}^{i}$ represents $i$-th realization of the independent random phase with uniform distributed probability density function between 0 and $2 \pi$.

As second step, it is needed to evaluate the response samples. In particular, for each input sample $F^{i}(t)$ we need to evaluate the output process $Y_{j}^{i}(t)$ in Eq. (47) with the aid of the Duhamel superposition integral in Eq. (49). Thus,

$$
Y_{j}^{i}(t)=\frac{c_{j}}{\rho A} \int_{0}^{t} h_{j}(t-\tau) F^{i}(\tau) d \tau
$$

As last step, after the evaluation of the $n \times N$ response processes $Y_{j}^{i}(t)$ with $i=1,2 \ldots, N$, and $j=1,2, \ldots, n$, the stochastic displacement process samples $v^{i}(z, t)$ can be used to evaluate the statistics numerically.

\subsection{Frequency domain approach}

Steady-state analysis and characterization of stationary responses can be driven in analytical way. Specifically, we make the truncated Fourier transform of the stochastic differential equation (47). That is,

$$
\hat{Y}_{j}(\omega, \mathrm{T})\left[-\omega^{2}+\frac{\eta}{\rho A} \mathrm{i} \omega+\frac{k_{\lambda, j}}{\rho A}\right]=\frac{c_{j}}{\rho A} \hat{F}(\omega, \mathrm{T})
$$


which transforms the differential equation in time domain to an algebraic equation in frequency domain. From Eq. (61) we get the solution $\hat{Y}_{j}(\omega, \mathrm{T})$ as

$$
\begin{aligned}
\hat{Y}_{j}(\omega, \mathrm{T}) & =\frac{1}{-\omega^{2} \hat{+} \frac{\eta}{\rho A} \mathrm{i} \omega+\frac{k_{\lambda, j}}{\rho A}} \frac{c_{j}}{\rho A} \hat{F}(\omega, \mathrm{T}) \\
& =H_{j}(\omega) \frac{c_{j}}{\rho A} \hat{F}(\omega, \mathrm{T})
\end{aligned}
$$

where $H_{j}(\omega)$ is the transfer function of the $j$-th modal oscillator. Once the response $\hat{Y}_{j}(\omega)$ in frequency domain is known, we can place it in the expression of the cross PSD of the response processes $Y_{j}(t)$ and $Y_{i}(t)$. That is,

$$
\begin{aligned}
S_{Y_{j} Y_{i}}(\omega) & =\lim _{\mathrm{T} \rightarrow \infty} \frac{1}{2 \pi \mathrm{T}} \mathbb{E}\left[\hat{Y}_{j}^{*}(\omega, \mathrm{T}) \hat{Y}_{i}(\omega, \mathrm{T})\right] \\
& =\frac{c_{j}}{\rho A} H_{j}^{*}(\omega) \lim _{\mathrm{T} \rightarrow \infty} \frac{1}{2 \pi \mathrm{T}} \mathbb{E}\left[\hat{F}^{*}(\omega, \mathrm{T}) \hat{F}(\omega, \mathrm{T})\right] \frac{c_{i}}{\rho A} H_{i}(\omega) \\
& =\frac{c_{j} c_{i}}{(\rho A)^{2}} H_{j}^{*}(\omega) H_{i}(\omega) S_{F}(\omega)
\end{aligned}
$$

Finally, by using Eq. (63), it is possible to evaluate the analytical form of the PSD of beam displacements as follows

$$
\begin{aligned}
S_{v}(z, \omega) & =\sum_{j=1}^{\infty} \sum_{i=1}^{\infty} \phi_{j}(z) \phi_{i}(z) \lim _{\mathrm{T} \rightarrow \infty} \frac{1}{2 \pi \mathrm{T}} \mathbb{E}\left[\hat{Y}_{j}^{*}(\omega, \mathrm{T}) \hat{Y}_{i}(\omega, \mathrm{T})\right] \\
& =\sum_{j=1}^{\infty} \sum_{i=1}^{\infty} \phi_{j}(z) \phi_{i}(z) S_{Y_{j} Y_{i}}(\omega) \\
& =\frac{S_{F}(\omega)}{(\rho A)^{2}} \sum_{j=1}^{\infty} \sum_{i=1}^{\infty} \phi_{j}(z) \phi_{i}(z) c_{j} c_{i} H_{j}^{*}(\omega) H_{i}(\omega)
\end{aligned}
$$

The PSD in Eq. (64) allows for evaluating the stationary variance of transverse displacements $\sigma_{v}^{2}(z)$. That is,

$$
\sigma_{v}^{2}(z)=\int_{-\infty}^{\infty} S_{v}(z, \omega) d \omega=\frac{1}{(\rho A)^{2}} \sum_{j=1}^{\infty} \sum_{i=1}^{\infty} \phi_{j}(z) \phi_{i}(z) c_{j} c_{i} \int_{-\infty}^{\infty} H_{j}^{*}(\omega) S_{F}(\omega) H_{i}(\omega) d \omega
$$

4.3 Nonlocal beam under Gaussian white noise

In this section we consider the case in which the input process is a Gaussian white noise with zero mean denoted by $W(t)$ and characterized by a constant PSD and a Dirac delta as CF. That is,

$$
S_{W}(\omega)=S_{0}, \quad R_{W}(\tau)=2 \pi S_{0} \delta(\tau)
$$

With this assumption the present study does not lose generality inasmuch several real excitation process can be modeled as summation of modulated white noises. 
Moreover, from this considered case analytical solutions in terms of statistics of the response can be obtained and some useful results about the time and frequency domain analysis can be drawn. Under the assumptions in Eq. (66), Eq. (64) and Eq. (65) provide the characterization of the stochastic output process in terms of PSD and stationary variance. Specifically,

$$
S_{v}(z, \omega)=\frac{S_{0}}{(\rho A)^{2}} \sum_{j=1}^{\infty} \sum_{i=1}^{\infty} \phi_{j}(z) \phi_{i}(z) c_{j} c_{i} H_{j}^{*}(\omega) H_{i}(\omega)
$$

and

$$
\sigma_{v}^{2}(z)=\frac{S_{0}}{(\rho A)^{2}} \sum_{j=1}^{\infty} \sum_{i=1}^{\infty} \phi_{j}(z) \phi_{i}(z) c_{j} c_{i} \int_{-\infty}^{\infty} H_{j}^{*}(\omega) H_{i}(\omega) d \omega
$$

However, the quantities in Eqs. (67) and (68) provide a characterization of the displacement just at steady-state. In order to provide a complete stochastic characterization of the response it is needed to evaluate the CF in Eq. (46) and the time-dependent non-stationary variance in Eq. (57). In this regard, exploiting the properties of the stochastic input, the $\mathrm{CF}$ of the displacement is given by

$$
R_{v}\left(z, t_{1}, t_{2}\right)=\sum_{j=1}^{\infty} \sum_{i=1}^{\infty} \phi_{j}(z) R_{Y_{j} Y_{i}}\left(t_{1}, t_{2}\right) \phi_{i}(z)
$$

where each term in the summations can be evaluated in closed form. That is,

$$
\begin{aligned}
R_{Y_{j} Y_{i}}\left(t_{1}, t_{2}\right) & =\frac{c_{j} c_{i} 2 \pi S_{0}}{(\rho A)^{2}} \int_{0}^{t_{1}} \int_{0}^{t_{2}} h_{j}\left(t_{1}-\tau_{1}\right) h_{i}\left(t_{2}-\tau_{2}\right) \delta\left(\tau_{2}-\tau_{1}\right) d \tau_{1} d \tau_{2} \\
& =\frac{c_{j} c_{i} 2 \pi S_{0}}{(\rho A)^{2}} \int_{0}^{t_{1}} h_{j}\left(t_{1}-\tau_{1}\right) h_{i}\left(t_{2}-\tau_{1}\right) d \tau_{1}
\end{aligned}
$$

From Eq. (69) the time-dependent variance of the displacement is

$$
\begin{aligned}
\sigma_{v}^{2}(z, t) & =R_{v}(z, t, t)=\sum_{j=1}^{\infty} \sum_{i=1}^{\infty} \phi_{j}(z) \phi_{i}(z) R_{Y_{j} Y_{i}}(t, t) \\
& =\frac{2 \pi S_{0}}{(\rho A)^{2}} \sum_{j=1}^{\infty} \sum_{i=1}^{\infty} c_{j} c_{i} \phi_{j}(z) \phi_{i}(z) \int_{0}^{t} h_{j}(t-\tau) h_{i}(t-\tau) d \tau
\end{aligned}
$$

Note that both the expressions in Eq. (70) and in Eq. (71) can be evaluated in analytical form.

\section{Numerical simulation and parametric study}

This section is devoted to the stationary and non-stationary analysis of the stochastic response of a nonlocal damped Bernoulli-Euler beam forced by a random load. Such numerical analysis aims to study the influence of the nonlocal parameter $\lambda$ in the response in terms of CF, PSD and stationary and non-stationary variance.

We consider a micro-beam of length $L=300 \mu \mathrm{m}$, rectangular cross section with width $b=30 \mu \mathrm{m}$ and thickness $h=25 \mu \mathrm{m}$, made of epoxy characterized by density $\rho=1.20 \mathrm{~g} / \mathrm{cm}^{3}$ and elastic modulus $E=2.20 \mathrm{GPa}[65,81]$. Damping effects 
due to surrounding environment are modeled by the following value of viscosity $\eta=2.00 \mathrm{cP}$ describing a wide variety of viscous fluids of technical interest $[81,82]$. The considered micro-beam is constrained as a cantilever beam and is forced by a ground motion acceleration as depicted in Fig. 3a. Without loss of generality, such imposed acceleration on the basis $z=0$ is a Gaussian white noise

$$
\ddot{v}(0, t)=a_{g}(t)=W(t)
$$

where the white noise $W(t)$ has zero-mean and constant PSD $S_{0}=10^{3} \mathrm{~N}^{2} \mathrm{~s}$, and $v(z, t)$ denotes the relative displacement with respect to the basis. In this case the partial differential equation (15) becomes

$\frac{\partial^{4} v(z, t)}{\partial z^{4}}-(\lambda L)^{2} \frac{\partial^{6} v(z, t)}{\partial z^{6}}+\frac{\eta}{E I} \frac{\partial v(z, t)}{\partial t}+\frac{\rho A}{E I}\left[\frac{\partial^{2} v(z, t)}{\partial t^{2}}+a_{g}(t)\right]=0, \quad 0<z<L$

and the BCs $\forall t$ are

$$
\begin{cases}v(0, t)=0, & M(L, t)=0, \\ \varphi(0, t)=0, & T(L, t)=0\end{cases}
$$

Let us recall that, by virtue Eq.(8) and Eq.(11), bending moment $M$ and shear force $T$ fields can be expressed in terms of elastic curvature and its derivatives as

$$
\begin{gathered}
M(z, t)=E I \chi(z, t)-E I(\lambda L)^{2} \frac{\partial^{2} \chi(z, t)}{\partial z^{2}} \\
T(z, t)=E I \frac{\partial \chi(z, t)}{\partial z}-E I(\lambda L)^{2} \frac{\partial^{3} \chi(z, t)}{\partial z^{3}}
\end{gathered}
$$

To show the effects of the nonlocal parameters in the response, different cases of Eq. (73) are considered. Specifically, we select three values of the nonlocal parameter $\lambda$, i.e., $\lambda=\{0.1,0.2,0.3\}$.

The solution in terms of displacement function is obtained with the aid of Eq. (38), where each eigenfunction is obtained by solving the differential problem in Eq. (22) with the two constitutive BCs in Eq. (23) and the four BCs in Eq. (74). The latter BCs in terms of eigenfunctions are

$$
\begin{cases}\phi(0)=0, & \phi^{(2)}(L)-(\lambda L)^{2} \phi^{(4)}(L)=0, \\ \phi^{(1)}(0)=0, & \phi^{(3)}(L)-(\lambda L)^{2} \phi^{(5)}(L)=0\end{cases}
$$

The first five eigenfunctions for $\lambda=0.2$ are shown in Fig. $3 \mathrm{~b}$ and the first five natural frequencies for different values of $\lambda$ are reported in Table 1 .

Taking Eq. (73) into account and with the aid of the definition in Eq. (67) the PSD of the displacement is

$$
S_{v}(z, \omega) \approx S_{0} \sum_{j=1}^{n} \sum_{i=1}^{n} \phi_{j}(z) \phi_{i}(z) c_{j} c_{i} H_{j}^{*}(\omega) H_{i}(\omega)
$$

where for the present numerical simulations we assume $n=5$.

In Fig. 4 the PSD of the displacements are reported for different values of $\lambda$. Specifically, Fig. 4a shows the PSDs of the mid-point displacements, while in Fig. 4b the PSDs at $z=L$ are shown. We can observe that the nonlocal parameter influences both the natural frequencies and the peak amplitudes in 


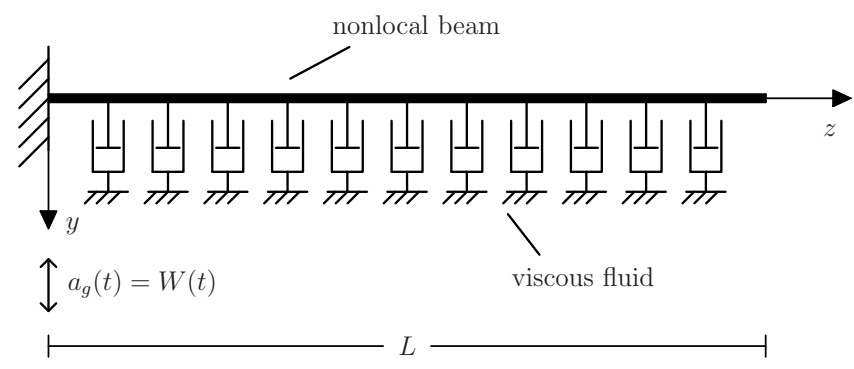

(a) Layout of the cantilever micro-beam

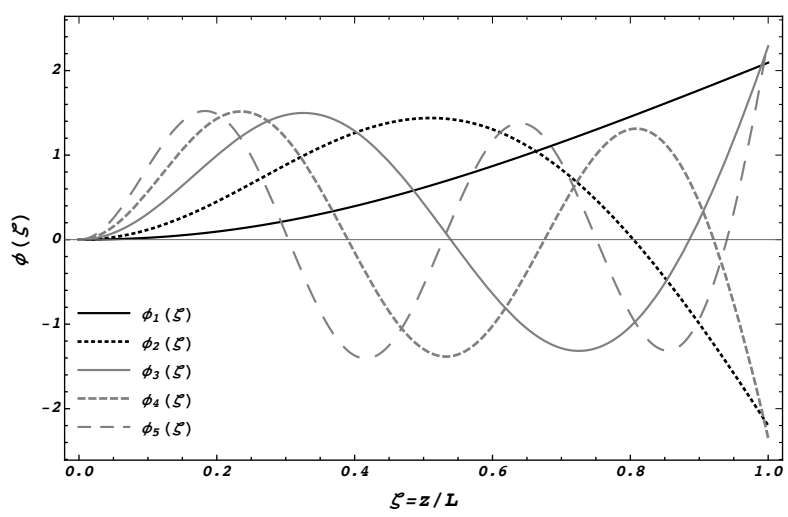

(b) First five eigenfunctions for $\lambda=0.2$

Fig. 3: Cantilever micro-beam forced by ground motion acceleration

\begin{tabular}{lccccc}
\hline$\lambda$ & $\omega_{0,1}$ & $\omega_{0,2}$ & $\omega_{0,3}$ & $\omega_{0,4}$ & $\omega_{0,5}$ \\
\hline 0.10 & $4.2323 \times 10^{5}$ & $2.8373 \times 10^{6}$ & $8.8780 \times 10^{6}$ & $1.9924 \times 10^{7}$ & $3.7820 \times 10^{7}$ \\
0.15 & $4.4551 \times 10^{5}$ & $3.1643 \times 10^{6}$ & $1.0609 \times 10^{6}$ & $2.5278 \times 10^{7}$ & $5.0090 \times 10^{7}$ \\
0.20 & $4.6795 \times 10^{5}$ & $3.5244 \times 10^{6}$ & $1.2495 \times 10^{7}$ & $3.1001 \times 10^{7}$ & $6.2992 \times 10^{7}$ \\
0.25 & $4.9002 \times 10^{5}$ & $3.9038 \times 10^{6}$ & $1.4464 \times 10^{6}$ & $3.6905 \times 10^{7}$ & $7.6186 \times 10^{7}$ \\
0.30 & $5.1192 \times 10^{5}$ & $4.2951 \times 10^{6}$ & $1.6481 \times 10^{7}$ & $4.2909 \times 10^{7}$ & $8.9539 \times 10^{7}$
\end{tabular}

Table 1: Natural frequencies in $\mathrm{rad} / \mathrm{s}$ of cantilever micro-beam for different values of $\lambda$.

the PSD. Specifically, when the nonlocal parameter increases the amplitude of the peaks decrease and their frequencies increases. From the PSD in Eq. (77) and with the aid of Eq. (65) we can also evaluate the stationary variance of the displacement. Such quantity can be evaluated by the integration of the PSD in Eq. (77) as follow

$$
\sigma_{v}^{2}(z)=\int_{-\infty}^{\infty} S_{v}(z, \omega) d \omega \approx S_{0} \sum_{j=1}^{n} \sum_{i=1}^{n} \phi_{j}(z) \phi_{i}(z) c_{j} c_{i} \int_{-\infty}^{\infty} H_{j}^{*}(\omega) H_{i}(\omega) d \omega
$$




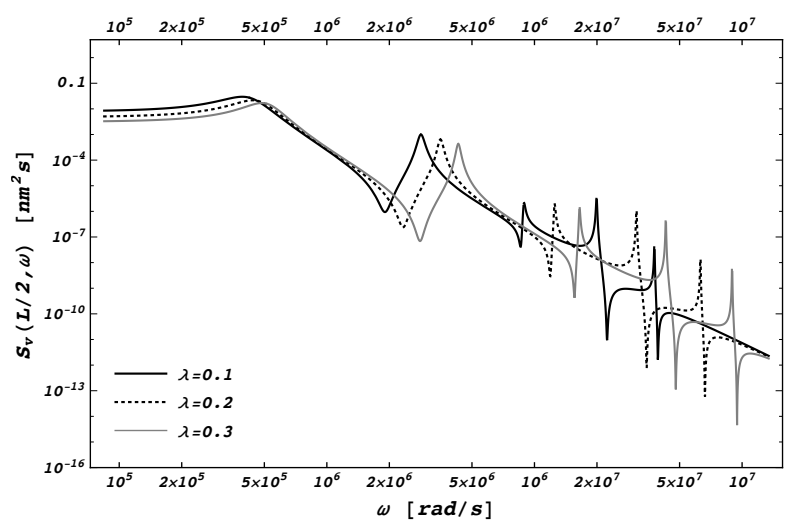

(a) PSD for $z=L / 2$

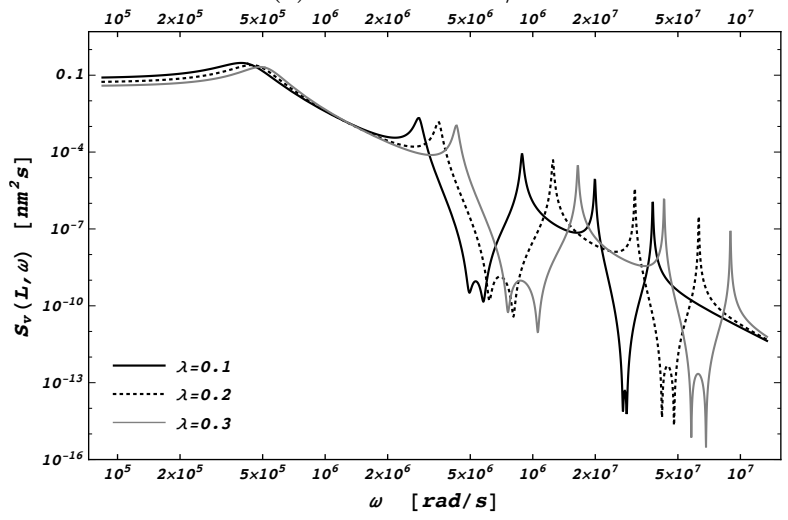

(b) PSD for $z=L$

Fig. 4: PSD of a cantilever micro-beam response for different values of $\lambda$

In Table 2 stationary variances at $z=L / 2$ and $z=L$ for different values of $\lambda$ are reported. Taking into account the values in this table we can state that the

\begin{tabular}{lcc}
\hline$\lambda$ & $\sigma_{v}^{2}(L / 2)$ & $\sigma_{v}^{2}(L)$ \\
\hline 0.10 & 0.0200 & 0.1975 \\
0.15 & 0.0165 & 0.1791 \\
0.20 & 0.0144 & 0.1645 \\
0.25 & 0.0138 & 0.1548 \\
0.30 & 0.0115 & 0.1416 \\
\hline
\end{tabular}

Table 2: Displacement variances in $\mu \mathrm{m}^{2}$ at $z=L / 2$ and $z=L$, for different values of $\lambda$. 
nonlocal parameter also influences stationary variances. Specifically, when the nonlocal parameter $\lambda$ grows up then the displacement stationary variances decrease.

The PSD in Eq. (77) and the stationary variance in Eq. (78) provide a steadystate characterization of the displacement process due to a stochastic ground motion acceleration. However, taking Eq. (73) into account, a characterization of the non-stationary response can be pursued by using Eq. (69) to evaluate the CF of the process $v(z, t)$. That is,

$$
R_{v}\left(z, t_{1}, t_{2}\right)=2 \pi S_{0} \sum_{j=1}^{\infty} \sum_{i=1}^{\infty} \phi_{j}(z) \phi_{i}(z) c_{j} c_{i} \int_{0}^{t_{1}} h_{j}\left(t_{1}-\tau_{1}\right) h_{i}\left(t_{2}-\tau_{1}\right) d \tau_{1}
$$

By virtue of the definition in Eq. (71) the time-dependent variance is

$$
\begin{aligned}
\sigma_{v}^{2}(z, t) & =R_{v}(z, t, t)=\sum_{j=1}^{\infty} \sum_{i=1}^{\infty} \phi_{j}(z) \phi_{i}(z) R_{Y_{j} Y_{i}}(t, t) \\
& =2 \pi S_{0} \sum_{j=1}^{\infty} \sum_{i=1}^{\infty} c_{j} c_{i} \phi_{j}(z) \phi_{i}(z) \int_{0}^{t} h_{j}(t-\tau) h_{i}(t-\tau) d \tau
\end{aligned}
$$

From Eq. (80) it is possible to obtain the stationary variance in Eq. (78) by performing the following limit

$$
\sigma_{v}^{2}(z)=\lim _{t \rightarrow \infty} \sigma_{v}^{2}(z, t)
$$

while the stationary $\mathrm{CF}$ is given by

$$
R_{v}(z, \tau)=\lim _{t \rightarrow \infty} R_{v}(z, t, t+\tau)
$$

the latter equation is related to the PSD in Eq. (77) by Fourier transform (WienerKhinchin theorem). Fig. 5 shows the stationary and non-stationary displacement variances for different values of the nonlocal parameter $\lambda$. Dashed lines represent the stationary variances, whereas continuous lines are the non-stationary ones obtained with the aid of Eq. (80). Such exact variances are compared with the numerical results obtained by means of the MC approach described in Section 4.1.1. Specifically, the numerical variances (dotted lines in Fig. 5) are obtained considering $N=4 \times 10^{3}$ samples and by assuming $m=2 \times 10^{3}$ and $\Delta \omega=\omega_{0,1} / 50$ in Eq. (59). From Fig. 5 we can observe that the steady state is reached in all considered case in a few tens of microseconds. This is due to the fact that the involved stiffnesses in such micro-beams are great and the masses are little. Moreover, the nonlocal parameter influences the duration of the transient state, indeed, if $\lambda$ grows up the variance reaches the stationary value more quickly.

\section{Concluding remarks}

Random vibrations of damped nonlocal Bernoulli-Euler beams due to stochastic excitation have been investigated in the present research. Two specific effects have been accurately analyzed: size and damping phenomena, respectively modeled by stress-driven nonlocal mechanics and external viscous interactions. A stochastic input for the loading has been assumed to simulate external actions randomness. 


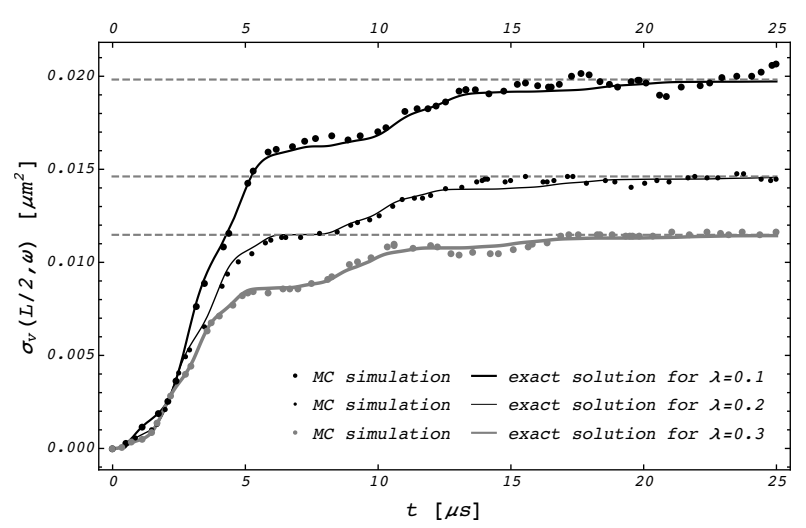

(a) Displacement variance at $z=L / 2$

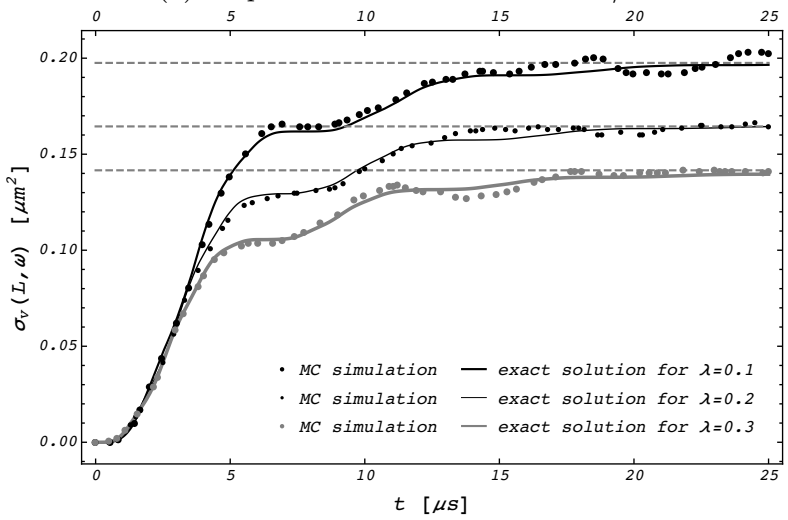

(b) Displacement variance at $z=L$

Fig. 5: Exact stationary (dashed line) and non-stationary (continuous line) displacement variance in contrast with those obtained by $\mathrm{MC}$ simulations

A stochastic differential problem in space and time, governing the motion of nonlocal beams under stochastic loading, has been formulated.

Exact solutions of power spectral density, correlation function and displacement variance have been evaluated by differential eigenanalysis.

From the analytical formulation of stationary and non-stationary responses and with the aid of numerical simulations, it has been highlighted a significant reduction of stationary variances and duration of the transient state in responses and an increasing of natural frequencies for increasing nonlocal scale parameter. The predicted smaller-is-stiffer phenomenon, confirmed recently in [49], agrees with most of experimental outcomes associated with inflected small-scale beams [83].

In summary, the nonlocal approach developed to model damped small-scale beams is able to capture size and damping effects and random excitations. The methodology provides analytical solutions in terms of statistics of the response and closed-form natural frequencies. The contributed results can be exploited for structural design and optimization of smaller and smaller devices used in modern technological applications, such as: sensors, actuators, MEMS/NEMS, resonators. 


\section{Acknowledgments}

Financial supports from the MIUR in the framework of the Projects PRIN 2015 "COAN 5.50.16.01" (code 2015JW9NJT Advanced mechanical modeling of new materials and structures for the solution of 2020 Horizon challenges) and PRIN 2017 (code 2017J4EAYB Multiscale Innovative Materials and Structures (MIMS); University of Naples Federico II Research Unit) and from the research program ReLUIS 2019 are gratefully acknowledged.

\section{Conflict of interest}

The authors declare that they have no conflict of interest.

\section{References}

1. Pourasghar A, Chen Z (2019) Effect of hyperbolic heat conduction on the linear and nonlinear vibration of CNT reinforced size-dependent functionally graded microbeams. International Journal of Engineering Science 137:57-72.

2. Xia X, Weng GJ, Hou D, Wen W (2019) Tailoring the frequency-dependent electrical conductivity and dielectric permittivity of CNT-polymer nanocomposites with nanosized particles. International Journal of Engineering Science 142:1-19.

3. Mojahedi M (2017) Size dependent dynamic behaviour of electrostatically actuated microbridges. International Journal of Engineering Science 111:74-85.

4. Moradweysi P, Ansari R, Hosseini K, Sadeghi F (2018) Application of modified Adomian decomposition method to pull-in instability of nano-switches using nonlocal Timoshenko beam theory. Applied Mathematical Modelling 54:594604 .

5. Hosseini SM (2018) Analytical solution for nonlocal coupled thermoelasticity analysis in a heat-affected MEMS/NEMS beam resonator based on GreenNaghdi theory, Applied Mathematical Modelling 57:21-36.

6. De Bellis ML, Bacigalupo A, Zavarise G (2019) Characterization of hybrid piezoelectric nanogenerators through asymptotic homogenization. Computer Methods in Applied Mechanics and Engineering 355:1148-1186.

7. Natsuki T, Urakami K (2019) Analysis of Vibration Frequency of Carbon Nanotubes used as Nano-Force Sensors Considering Clamped Boundary Condition. Electronics 8(10):1082.

8. Mohammadian M, Abolbashari MH, Hosseini S.M (2019) Application of hetero junction CNTs as mass nanosensor using nonlocal strain gradient theory: An analytical solution. Applied Mathematical Modelling 76:26-49.

9. Tran N, Ghayesh MH, Arjomandi M (2018) Ambient vibration energy harvesters: A review on nonlinear techniques for performance enhancement. International Journal of Engineering Science 127:162-185.

10. Basutkar R (2019) Analytical modelling of a nanoscale series-connected bimorph piezoelectric energy harvester incorporating the flexoelectric effect. International Journal of Engineering Science 139:42-61. 
11. Ghayesh MH, Farokhi H (2020) Nonlinear broadband performance of energy harvesters. International Journal of Engineering Science 147:103202.

12. Farajpour A, Ghayesh MH, Farokhi H (2018) A review on the mechanics of nanostructures. International Journal of Engineering Science 133:231-263.

13. Bauer S, Pittrof A, Tsuchiya H, Schmuki P (2011) Size-effects in TiO(2) nanotubes: diameter dependent anatase/rutile stabilization. Electrochemistry Communications 13:538-541.

14. Kiang C, Endo M, Ajayan P, Dresselhaus G, Dresselhaus M (1998) Size effects in carbon nanotubes. Physical Review Letters 81:1869-1872.

15. Xiao S, Hou W (2006) Studies of size effects on carbon nanotubes' mechanical properties by using different potential functions. Fullerenes Nanotubes and Carbon Nanostructures 14:9-16.

16. Zienert A, Schuster J, Streiter R, Gessner T (2010) Transport in carbon nanotubes: contact models and size effects. Physica Status Solidi B-basic Solid State Physics 247:3002-3005.

17. Chowdhury R, Adhikari S, Wang C, Scarpa F (2010) A molecular mechanics approach for the vibration of single-walled carbon nanotubes. Computational Materials Science 48:730-735.

18. Tang C, Meng L, Sun L, Zhang K, Zhong J (2008) Molecular dynamics study of ripples in graphene nanoribbons on $6 \mathrm{H}-\mathrm{SiC}(0001)$ : temperature and size effects. Journal of Applied Physics 104. Paper 113536.

19. Marotti de Sciarra F (2009) A nonlocal model with strain-based damage. International Journal of Solids and Structures 46(22-23):4107-4122.

20. Wang LF, Hu HY (2005) Flexural wave propagation in single-walled carbon nanotube. Physical Review B 71(19):195412-195418.

21. Lu P, Lee HP, Lu C, Zhang PQ (2007) Application of nonlocal beam models for carbon nanotubes. International Journal of Solids and Structures 44(16):52895300 .

22. Alotta G, Failla G, Zingales M (2014) Finite element method for a nonlocal Timoshenko beam model. Finite Element in Analysis and Design 89:77-92.

23. Marotti de Sciarra F (2014) Finite element modelling of nonlocal beams. Physica E: Low-Dimensional Systems and Nanostructures 59:144-149.

24. Ansari R., Sahmani S, Arash B (2010) Nonlocal plate model for free vibrations of single-layered graphene sheets. Physics Letters A 375:53-62.

25. Murmu T, Adhikari S (2011) Nonlocal vibration of carbon nanotubes with attached buckyballs at tip. Mechanics Research Communications 38:62-67.

26. Ansari R, Sahmani S (2012) Small scale effect on vibrational response of singlewalled carbon nanotubes with different boundary conditions based on nonlocal beam models. Communications in Nonlinear Science and Numerical Simulation 17:1965-1979.

27. Lakes RS (1991) Experimental micro mechanics methods for conventional and negative Poissons ratio cellular solids as Cosserat continua. Journal of Engineering Materials and Technology 113(1):148-155.

28. Arash B, Wang Q (2012) A review on the application of nonlocal elastic models in modeling of carbon nanotubes and graphenes. Computational Materials Science 51(1):303-313.

29. Rogula D (1965) Influence of spatial acoustic dispersion on dynamical properties of dislocations. Bull Acad Pol Sci Ser Sci Tech 13:337-343. 
30. Rogula D (1982) Introduction to nonlocal theory of material media. In: Nonlocal theory of material media. CISM courses and lectures, Rogula D, ed., Springer, Wien, 268:125-222.

31. Eringen AC (1972) Linear theory of nonlocal elasticity and dispersion of plane waves. International Journal of Engineering Science 10:425-435.

32. Eringen AC (1983) On differential equations of nonlocal elasticity and solutions of screw dislocation and surface waves. Journal of Applied Physics 54:4703.

33. Tricomi FG (1957) Integral Equations. Interscience, New-York, USA. Reprinted by Dover Books on Mathematics, 1985.

34. Polyanin AD, Manzhirov AV (2008) Handbook of integral equations. 2nd ed. Boca Raton, FL: Chapman \& Hall/CRC.

35. Romano G, Barretta R, Diaco M, Marotti de Sciarra F (2017) Constitutive boundary conditions and paradoxes in nonlocal elastic nano-beams. Int J Mech Sci 121:151-156.

36. Challamel N, Wang CM (2008) The small length scale effect for a non-local cantilever beam: a paradox solved. Nanotechnology 19:345703.

37. Fernández-Sáez J, Zaera R, Loya JA, Reddy JN (2016) Bending of EulerBernoulli beams using Eringen's integral formulation: A paradox resolved. International Journal of Engineering Science 99:107-116.

38. Borino G, Failla B, Parrinello F (2003) A symmetric nonlocal damage theory. International Journal of Solids and Structures 40:3621-3645.

39. Khodabakhshi P, Reddy JN (2015) A unified integro-differential nonlocal model. International Journal of Engineering Science 95:60-75.

40. Lam DCC, Yang F, Chong ACM, Wang J, Tong P (2003) Experiments and theory in strain gradient elasticity. Journal of Mechanics and Physics of Solids 51(8):1477-1508.

41. Challamel N, Reddy JN, Wang CM (2016) Eringen's stress gradient model for bending of nonlocal beams. Journal of Engineering Mechanics 142(12):04016095.

42. Numanoğlu HM, Akgöz, B., Civalek Ö (2018) On dynamic analysis of nanorods. International Journal of Engineering Science 130:33-50.

43. Cornacchia F, Fantuzzi N, Luciano R, Penna R (2019) Solution for cross- and angle-ply laminated Kirchhoff nano plates in bending using strain gradient theory. Composites Part B: Engineering 173:107006.

44. Cornacchia F, Fabbrocino F, Fantuzzi N, Luciano R, Penna R (2019). Analytical solution of cross- and angle-ply nano plates with strain gradient theory for linear vibrations and buckling, Mechanics of Advanced Materials and Structures, DOI: 10.1080/15376494.2019.1655613

45. Lim CW, Zhang G, Reddy JN (2015) A higher-order nonlocal elasticity and strain gradient theory and its applications in wave propagation. Journal of the Mechanics and Physics of Solids 78:298-313.

46. Barretta R, Marotti de Sciarra F (2018) Constitutive boundary conditions for nonlocal strain gradient elastic nano-beams. International Journal of Engineering Science 130:187-198.

47. Apuzzo A, Barretta R, Faghidian SA, Luciano R, Marotti de Sciarra F (2018) Free vibrations of elastic beams by modified nonlocal strain gradient theory. International Journal of Engineering Science 133:99-108. 
48. Polizzotto C, Fuschi P, Pisano AA (2004) A strain-difference-based nonlocal elasticity model. International Journal of Solids and Structures 41:2383-2401.

49. Fuschi P, Pisano AA, Polizzotto C (2019) Size effects of small-scale beams in bending addressed with a strain-difference based nonlocal elasticity theory. International Journal of Mechanical Sciences 151:661-671.

50. Marotti de Sciarra F (2009) On non-local and non-homogeneous elastic continua. International Journal of Solids and Structures 46(3-4):651-676.

51. Di Paola M, Zingales M (2008) Long-range cohesive interactions of non-local continuum faced by fractional calculus. International Journal of Solids and Structures 45:5642-5659.

52. Di Paola M, Pirrotta A, Zingales M (2010) Mechanically-based approach to non-local elasticity: Variational principles. International Journal of Solids and Structures 47(5):539-548.

53. Di Paola M, Failla G, Zingales M (2013) Non-local stiffness and damping models for shear-deformable beams. European Journal of Mechanics A/Solids 40:69-83.

54. Failla G, Sofi A, Zingales M (2015) A new displacement-based framework for non-local Timoshenko beams. Meccanica 50(8):2103-2122.

55. Romano G, Barretta R (2017) Nonlocal elasticity in nanobeams: the stressdriven integral model. International Journal of Engineering Science 115:14-27.

56. Barretta R, Marotti de Sciarra F, Vaccaro MS (2019) On nonlocal mechanics of curved elastic beams. International Journal of Engineering Science 144:103140 .

57. Romano G, Barretta R (2017) Stress-driven versus strain-driven nonlocal integral model for elastic nano-beams. Composites Part B: Engineering 114:184188.

58. Barretta R, Čanadija M, Feo L, Luciano R, Marotti de Sciarra F, R. Penna (2018) Exact solutions of inflected functionally graded nano-beams in integral elasticity, Composites Part B 142:273-286.

59. Apuzzo A, Barretta R, Luciano R, Marotti de Sciarra F, Penna R (2017) Free vibrations of Bernoulli-Euler nano-beams by the stress-driven nonlocal integral model. Composites Part B: Engineering 123:105-111.

60. Lee J, Lin C (2010) The magnetic viscous damping effect on the natural frequency of a beam plate subject to an in-plane magnetic field. Journal of Applied Mechanics 77. Paper 011014.

61. Chen C, Ma M, Liu J, Zheng Q, Xu Z (2011) Viscous damping of nanobeam resonators: humidity, thermal noise, and a paddling effect. Journal of Applied Physics 110. Paper 034320.

62. Di Paola M, Fiore V, Pinnola FP, Valenza A (2014) On the influence of the initial ramp for a correct definition of the parameters of the fractional viscoelastic material. Mechanics of Materials 69:63-70.

63. Di Mino G, Airey G, Di Paola M, Pinnola FP, D'Angelo G, Lo Presti D (2016) Linear and nonlinear fractional hereditary constitutive laws of asphalt mixtures. Journal of Civil Engineering and Management 22(7):882-889.

64. Calleja M, Kosaka P, San Paulo A, Tamayo J (2012) Challenges for nanomechanical sensors in biological detection. Nanoscale 4:4925-4938.

65. T. Baidyk et al. (2005) MEMS/NEMS. Handbook techniques and applications. Edited by CT Leondes, University of California, Los Angeles, USA. 
66. Verma VK, Yadava RDS (2016) Stochastic resonance in MEMS capacitive sensors. Sensors and Actuators B: Chemical 235:583-602.

67. Roberts JB, Spanos PD (1999) Random vibrations and statistical linearization. Dover Publication, Inc., New-York, USA.

68. Crandall SH,Mark WD (1963) Random Vibration in Mechanical Systems. Academic Press, Inc., New-York, USA.

69. Di Paola M, Pirrotta A (1999) Non-linear systems under impulsive parametric input. International Journal of Non-Linear Mechanics 34(5):843-851.

70. Pirrotta A (2005) Non-linear systems under parametric white noise input: Digital simulation and response. International Journal of Non-Linear Mechanics 40(8):1088-1101.

71. Alotta G, Di Paola M, Pinnola FP (2017) Cross-correlation and cross-power spectral density representation by complex spectral moments. International Journal of Non-Linear Mechanics 94:20-27.

72. Lei Y, Murmu T, Adhikari S, Friswell MI (2013) Dynamic characteristics of damped viscoelastic nonlocal Euler-Bernoulli beams. European Journal of Mechanics A/Solids 42:125-136.

73. Lei Y, Adhikari S, Friswell MI (2013) Vibration of nonlocal Kelvin-Voigt viscoelastic damped Timoshenko beams. International Journal of Engineering Science 66-67:1-13.

74. Alotta G, Failla G, Pinnola FP (2017) Stochastic Analysis of a Nonlocal Fractional Viscoelastic Bar Forced by Gaussian White Noise. ASCE-ASME J. of Risk and Uncertainty in Engineering Systems, Part B: Mechanical Engineering 3(3):030904-030904-7.

75. Alotta G, Di Paola M, Failla G, Pinnola FP (2018) On the dynamics of nonlocal fractional viscoelastic beams under stochastic agencies. Composites Part B: Engineering 137:102-110.

76. Pirrotta A, Cutrona S., Di Lorenzo S, Di Matteo A (2015) Fractional viscoelastic Timoshenko beam deflection via single equation. International Journal for Numerical Methods in Engineering 104:869-886.

77. Alotta G, Failla G, Zingales M (2017) Finite element formulation of a nonlocal hereditary fractional order Timoshenko beam. Journal of Engineering Mechanics - ASCE 143(5): 1943-7889.0001035.

78. L. Meirovitch (2001) Fundamentals of Vibrations. McGraw-Hill International Edition.

79. Di Lorenzo S, Di Paola M, Pinnola FP, Pirrotta A (2014) Stochastic response of fractionally damped beams, Probabilistic Engineering Mechanics 35:37-43.

80. Shinozuka M, Deodatis G (1988) Stochastic process models for earthquake ground motion. Probabilistic Engineering Mechanics 3(3):114-123.

81. Ashby M, Shercliff H, Cebon D (2007) Materials engineering, science, processing and design. Edited by Elsevier, Burlington, USA.

82. Ashby M (1999) Materials selection in mechanical design. Edited by Butterworth-Heinemann, Woburn, USA.

83. Abazari AM, Safavi SM, Rezazadeh G, Villanueva LG (2015) Modelling the Size Effects on the Mechanical Properties of Micro/Nano Structures. Sensors 15:28543-28562. 